\title{
Identification and Pathogenic Potential of Orofacial Herpetic Clinical Isolates in Northeast Mexico
}

\author{
Cynthia Mendoza-Rodriguez ${ }^{1}$, Jorge Ocampo-Candiani ${ }^{1}$, Pilar Morales-San Claudio ${ }^{2}$, \\ Osvaldo Vazquez-Martinez ${ }^{1}$, Mauricio Salinas-Santander ${ }^{3}$, Ernesto Torres-Lopez ${ }^{4,}$ * \\ ${ }^{1}$ Dermatology Service, Faculty of Medicine and Dr. Jose Eleuterio Gonzalez University Hospital, Autonomous University of Nuevo Leon, \\ Nuevo León, Mexico \\ ${ }^{2}$ Microbiology Department, Center of Specialized Laboratories, Autonomous University of Nuevo Leon, Nuevo Leon, Mexico \\ ${ }^{3}$ Research Department, Faculty of Medicine, Autonomous University of Coahuila, Saltillo Coahuila, Mexico \\ ${ }^{4}$ Immunovirology Laboratory, Department of Immunology, Faculty of Medicine, Autonomous University of Nuevo Leon, Nuevo Leon, \\ Mexico
}

\author{
Email address: \\ ernestotorreslopez57@gmail.com (E. Torres-López) \\ ${ }^{*}$ Corresponding author
}

\section{To cite this article:}

Cynthia Mendoza-Rodriguez, Jorge Ocampo-Candiani, Pilar Morales-San Claudio, Osvaldo Vazquez-Martinez, Mauricio Salinas-Santander, Ernesto Torres-Lopez. Identification and Pathogenic Potential of Orofacial Herpetic Clinical Isolates in Northeast Mexico. International Journal of Clinical and Experimental Medical Sciences. Vol. 6, No. 5, 2020, pp. 91-95. doi: 10.11648/j.ijcems.20200605.12

Received: September 18, 2020; Accepted: October 5, 2020; Published: October 13, 2020

\begin{abstract}
Background Herpes simplex viruses 1 (human herpes virus types 1, HSV-1) often cause recurrent infections that affect the skin, mouth, lips, and eyes and eventually induce herpetic encephalitis. A high percentage of the population is infected with HSV-1 in which it produces a variety of these orofacial disease. In Mexico, there are no studies to determine the effects of viral virulence of clinical facial dermal isolates of active infections caused by the Herpes simplex virus 1. Objective of this work was to compare the herpetic activity of human clinical isolates from northeast Mexico against HSV-1 KOS as reference strain, which induces experimental murine model keratitis disease produced by infecting mouse corneas. Methods and Materials we compared several clinical isolate of HSV-1 obtained from 25 patients diagnosed with HSV-1 active, according to acyclovir (ACV) susceptibility, thymidine kinase (TK) polymerase chain reaction (PCR), and experimental Balb/c mice model as viral infections in vivo were evaluated. Results we found that several clinical isolates showed ACV resistance (48\%) and pathogenic potential (PP) differences that caused ocular infection more or less than reference HSV-1 KOS strain. In Conclusion, some clinical isolate from northeast Mexico shown differences that caused ocular infection more or less than reference HSV-1 KOS strain.
\end{abstract}

Keywords: Herpes Simplex Virus, Virulence, Experimental Model, Clinical Isolates, Acyclovir, Northeast Mexico

\section{Introduction}

Herpes viruses are approximately around $150 \mathrm{~nm}$ virions that possess an envelope containing glycoproteins [1], with a DNA center surrounded by an icosahedral capsid whose replication occurs in the nucleus at the intracellular level and belong to the group of Alphaherpesvirus [2]. Herpes simplex viruses 1 (HSV-1) infection is the most common cause of orolabial belong to the family of Herpesviridae, causing oral herpes lesions, meningitis, herpetic keratitis (a viral infection of the eye, also known as herpetic keratoconjunctivitis) and grave case encephalitis [3]. Much attention has been focused on host factors in determining these disease patterns and the differences among or pathogenesis virus strain within a single type that may account for the variation in activity herpetic ocular disease; however, in Mexico there are no studies in this regard [4]. Some HSV strains appear to produce specific disease patterns in the mouse cornea that simulate the various forms of human herpetic ocular infections [4]. Based on work by Irvine and Kimura, studied experimental disciform edema and necrotizing stromal keratitis model in the rabbit cornea by direct intrastromal inoculation with the strain of HSV [5]. However, numerous patients with immunosuppression often suffer relapses. These increase the emergence of strains 
resistant to first-line drugs as ACV. Also, it is known that herpes simplex is associated with the development of herpetic encephalitis particularly in old person [6], and it has been hypothesized that it can produce Alzheimer's disease, although the mechanism are not yet fully elucidated [7]. This denotes the importance of research any pathogenic activity versus antiviral resistance drug. On the other hand Wang $\mathrm{H}$ et $a l$. , has described that it has been observed that strains of HSV-1 vary in their pathogenesis [8]. In addition, viral recombination can be clinically significant; the high worldwide seroprevalence of HSV's strains raises the possibility of recombination between these viruses and wild-type ones [9]. Recently Colgrove et al., reported a strain SD90 (HSV-2) high virulence clinical isolate [10], more than HSV-2G, which in recent decades has been taken as reference strain to genital herpetic infection. Although host factors are important, previous laboratory experience suggests that the different clinical patterns of herpetic ocular disease may be attributed at least partially to the differing biological behavior and virulence of specific strains. $[10,11]$ For this reason and because the pathogenicity is multifactorial and depends on the immune status of the host as well as the virus that infects and in turn the nature of the viral strain in addition to its virulence factors, the aim of these work was to compare the herpetic activity of clinical isolates from north-east Mexico against HSV-1 KOS as reference strain, that induces experimental murine model keratitis disease produced by infecting mouse corneas.

\section{Materials and Methods}

\subsection{Clinical Isolates and Patients}

We performed an observational study, which recruited patients attending the Dermatology department of the "Dr. Jose Eleuterio Gonzalez" University Hospital diagnosed with HSV-1 active, during November 2009 to November 2010, who wished to participate voluntarily after informed consent was signed. Patients were asked about comorbidities, history of use of antivirals or if they were using them at the time, if they had previously had HSV infections, the number of episodes per year and infection recurrence at one year after sampling. The Ethics and Research Committee-Universidad Autónoma de Nuevo León approved and registered this study under the code INV-008 and INV-15001.

For sampling, the base of the vesicle was scraped with a Calcium Alginate Swabs (Fisher Scientific de Mexico, S. A., Monterrey, Mexico), obtaining approximately $0.3 \mathrm{~mL}$ of vesicular serous fluid, which was later introduced in $1 \%$ glucose solution for storage.

\subsection{Virus Typing}

Infection of a monolayer of Vero cells (ATCC $\AA$ CCL-81 $11^{\mathrm{TM}}$ ), was performed in T25 culture flasks at a confluence of $90 \%$. T25 flasks were incubated at $37^{\circ} \mathrm{C}$ whit stirring rotation at 250 rpm for 1 hour; $0.5 \mathrm{ml}$ of Advanced-DMEM 5\% FCS (Gibco BRL, Grand Island, NY, USA.) culture medium was then added and allowed to incubate at $37^{\circ} \mathrm{C} / 5 \% \mathrm{CO} 2$ and $10 \%$ humidity for 72 hours to observe cytopathic damage. Finally, cells infected with the isolated virus were harvested and stored in $1 \mathrm{~mL}$ aliquots at $-80^{\circ} \mathrm{C}$. In all isolates, $\mathrm{PCR}$ was performed to confirm HSV using the thymidine kinase (TK) gene. Starting with the positive samples in cell culture, samples were thawed gradually placing these in $175 \mathrm{~cm}_{2}$ flasks with confluent Vero cells for harvesting until $90 \%$ infection was seen. Aliquots were made and stored at $-80^{\circ} \mathrm{C}$ until use. We worked with a reference strain obtained from the ATCC (VR ${ }^{\circledR}-1493^{\mathrm{TM}}$ ), which corresponded to the HSV-1KOS strain. A $0.5 \mathrm{~mL}$ vial of the isolate was taken for amplification, performing serial 10 logarithmic dilutions from $10^{\mathrm{e}-1}$ to $10^{\mathrm{e}-10}$; dilutions were performed in a PBS/glucose buffer solution. After performing each of these dilutions, they were placed in 6-well plates containing $5 \times 105$ Vero cells, incubated at $37^{\circ} \mathrm{C}$, stirring at $250 \mathrm{rpm}$ for one hour. Subsequently, the viral suspension was discarded and reconstituted with a new Advanced-DMEM culture medium. The culture plates were subsequently incubated under the same conditions previously described for 72 hours. Subsequently, they were removed from incubation, stripped of culture medium, and then subjected to Giemsa staining. Finally, we determined the amount of plaque-forming units $(\mathrm{PFU}) / \mathrm{mL}$ and the viral titer.

According to the viral titers were adjusted to a concentration thereof of each of the samples was adjusted to a concentration of $50 \mathrm{PFU} / 0.5 \mathrm{~mL}$. For this assay culture plates containing 12 wells with $3 \times 10^{5}$ cells, each was made. Simultaneously, the ACV dilution was carried out; for this were a maximum concentration of $6.25 \mu \mathrm{g} / \mathrm{mL}$ and a minimum of $0.012 \mu \mathrm{g} / \mathrm{mL}$, afterward, the virus was added to a concentration of $50 \mathrm{PFU}$ to the 12 wells with Vero cells, letting these incubate at $37^{\circ} \mathrm{C}$ under gentle stirring for 1 hour. The viral suspension was withdrawn and reconstituted with Advanced-DMEM culture medium with $0.32 \% \operatorname{IgG}[12,13]$. ACV was added at the concentrations described before. The culture plates were subsequently incubated under the same conditions previously described for 72 hours. The $50 \%$ inhibitory concentration (IC50) was determined for each sample. A sample was considered resistant if the IC50 was found at a concentration $>3.1 \mu \mathrm{g} / \mathrm{mL}$ for ACV (3).

\subsection{DNA Isolation and PCR}

The vial containing the patient sample was mixed by vortex transferring the entire contents into a sterile micro tube, which was centrifuged at $12000 \mathrm{rpm}$ for 7 minutes; the cell pellet was resuspended in lysis solution. Columns of total DNA Isolation System (Wizard ${ }^{\circledR}$, Promega Corporation, Madison, WI, USA) were used to obtain and purify genetic material, following the manufacturer's instructions. The DNA was precipitated and finally resuspended in $20 \mu \mathrm{L}$ of Rehydration solution. This was stored at $-20^{\circ} \mathrm{C}$ until use.

For conducting PCR we used $4 \mu \mathrm{L}$ of the sample and $16 \mu \mathrm{L}$ of the reaction mixture, which contains the primer against the TK gene of HSV-1, was used to detect the effect TK gene of HSV-1 KOS as control positive, and $0.5 \mu \mathrm{M}$ of each primer: 5'-GGTGGGGAAAAGGAAGAA-3', as hsv1 tkir (internal reverse) and 5'-TAACAGCGTCAACAGCGT-3' as hsv1 tkif 
(internal forward) sequences (Integrated DNA Technologies, IDT), $0.2 \mathrm{mM}$ dNTPs, $2 \mathrm{mM} \mathrm{MgCl} 2$ and 1 units of Taq DNA polymerase (Go Taq Flexi, Promega Corporation, Madison, WI, USA). The PCR conditions were carried out initial denaturation at $94^{\circ} \mathrm{C}$ for 5 minutes, and 30 cycles of denaturation at $94^{\circ} \mathrm{C}$ for 30 seconds, annealing at $54^{\circ} \mathrm{C}$ for 30 seconds and elongation at $72^{\circ} \mathrm{C}$ for 30 seconds. The generated PCR fragments were analyzed by electrophoresis on $1 \%$ agarose gel stained with ethidium bromide and analyzed in a gel documentation system (UVP model M 26; UVP, LLC, Upland, CA, USA). A product of approximately $250 \mathrm{bp}$ was expected. Assays for DNA were performed separately for each clinical isolate to be amplified, using a portion of the DNA. All of them were reported as positive or negative to the obtaining of an amplification product on agarose gel electrophoresis.

\subsection{The Experimental Model of Herpetic Infection}

We used 8 week-old and $25 \mathrm{~g}$ weight male Balb/c mice (Harlan Co, Ind, USA). Animals were maintained four per case and under normal conditions with Purina rodent food and water available at libitum. The Animal Care and Use Committee approved animal housing and all procedures involving mice in accordance with federal guidelines. Requirements for care and handling of experimental animals according to international and Mexican regulations (IN-015 001, NOM-062-Z00-1999) were met. Male mice were infected as described previously $(12,13)$, with minor changes; each mouse was anesthetized with $85 \mathrm{mg}$ sodium pentobarbital $/ \mathrm{kg}$ of body weight (Nembutal; Abbott Laboratories) or a mixture delivering $100-\mathrm{mg} / \mathrm{kg}$ ketamine and $7 \mathrm{mg} / \mathrm{kg}$ Xylazine (Sigma-Aldrich, St. Louis, MO, USA). Each mouse was infected with 2 x106 PFU/eye; the virus was dropped onto both eyes in a $5-\mu$ Lvolumeeach. From the eye, the infection was a daily record using clinical observation and assessment of injuries through the viral keratitis eye. Each eye received separately a scale of severity (scale of 0 to 4) according to the following scale clinic and evaluation criteria in the ocular injury by infection of HSV-1, previously established: $0=$ cornea normal, $0.5=$ injury puntatas, $1.0=$ dendritic injuries, $1.5=$ stromal edema with geographic lesions, $2.0=$ stromal involvement, pupils and visible iris, $3.0=$ stromal involvement, pupils and iris invisible and $4.0=$ severe keratitis pupils and iris invisible [13]

\section{Results}

A total of 25 patients were included infected with HSV-1. The average age of the sample was 31.6 years. A history of HSV infection was present in $85.7 \%$ of the patients; on average, patients had 4.5 episodes per year. Of the group of patients with HSV-1, 22 (88\%) were immunocompetents, 3 (12\%) were immunosuppressed; on the other hand, of the 25 patient sonly 1 (4\%) had acute lymphoblastic leukemia and $2(8 \%)$ had autoimmune disease: idiopathic thrombocytopenic purpura and systemic lupus erythematous, respectively. All them with herpetic active infection with HSV-1 and $84 \%$ referred having been previously infected with HSV-1, while four patients (16\%) denied having had a previous herpes infection.

The analysis carried out by virus susceptibility test allowed to determine that fourteen patients with HSV-1 infection $(48 \%)$ had a positive viral culture; all had a viral titer greater than the reference strain.

On the other hand when submitting the total samples analyzed in this work to PCR for viral TK, only 11 samples (44\%) resulting positive; 10 were positive to culture and one negative for the viral culture. The product of amplification for the TK gene was expected to be approximately $250 \mathrm{bp}$. The determination of susceptibility to ACV performed by plaque reduction assay for the clinical isolates with a positive viral culture, showed an IC50 with a minimum value of $0.01 \mu \mathrm{g} / \mathrm{mL}$ and a maximum value of $1.46 \mu \mathrm{g} / \mathrm{mL}$ with a median of 0.150 $\mu \mathrm{g} / \mathrm{mL}$, therefore no isolate was resistant to ACV. Thirty-three percent of the patients with a positive viral culture had clinical recurrence at one-year follow up with an average of 1.6 episodes per year.

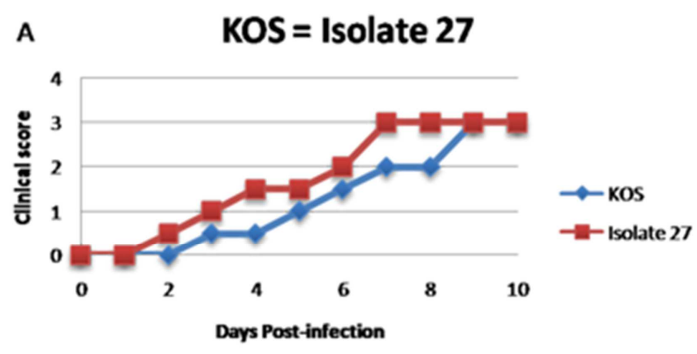

B Isolates more pathogenic than KOS

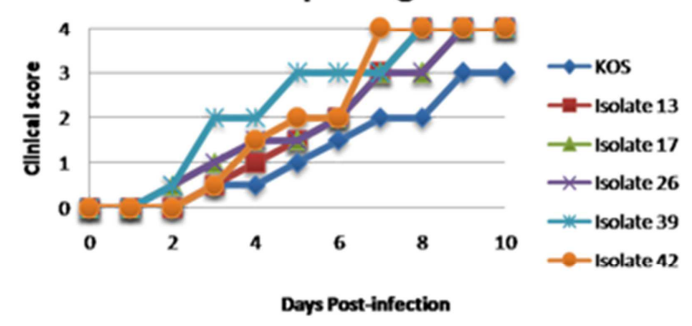

c Isolates less pathogenic than KOS

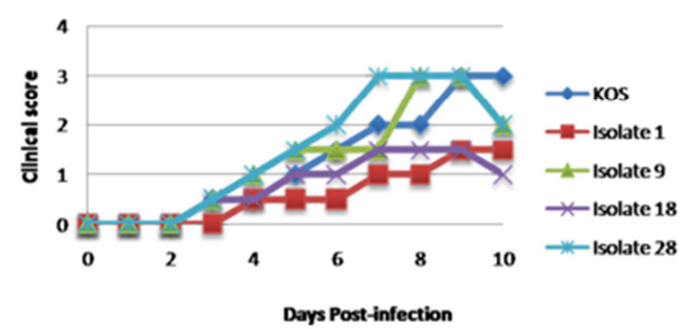

Figure 1. Differential pathogenesis activity patterns with HSV-1 clinical isolate. (A) BALB/c mice were KOS infected or infected with $5 \times 10^{6} \mathrm{PFU}$ of isolate 27. (B) BALB/c mice were KOS infected or infected with $5 \times 10^{6} \mathrm{PFU}$ of $13,17,26,39$ and 42 isolate. (C) BALB/c mice were KOS infected or infected with $5 \times 10^{6} \mathrm{PFU}$ of 1, 9, 18 and 28 isolate. (n=4). Pathogenesis as clinical score the mean is shown $(n=4)$, at days 2-3, (A) values for HSV-1 KOS were significantly lower $(P<0.05$, ANOVA) than those of other isolate and all them isolates were similar as HSV1 KOS (A) but significantly high (B) and lower $(C)(P<0.05$, ANOVA) than those of other HSV1 KOS strain; at days 9-10, values for HSV1 KOS strain were significantly lower $(P<0.05$, ANOVA) than those of other isolates. 


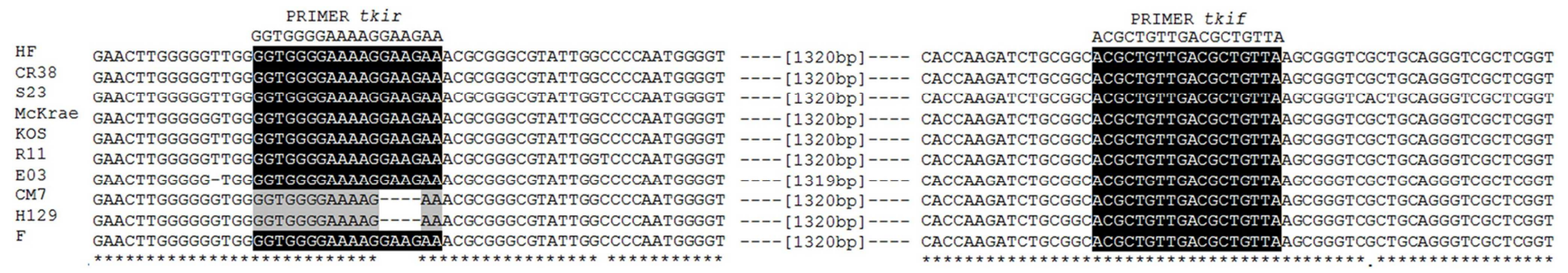

Figure 2. Thymidine kinase sequence alignment of different HSV-1 strains and tk primers. As shown in the figure, tkir primer shows lack of recognition with some HSV-1 variants similar to the H129 strain. The numbers in the center indicate the number of base pairs (bp) between the primers of the aligned sequences, respectively.

We chose a mouse model of ocular infection with HSV-1 KOS strain because the inbred mouse model has been provides a large number of viral model infection [13]. Ocular inoculation of $2 \times 10^{6}$ PFU of KOS or isolate viruses by this protocol resulted in keratoconjuntivitis in the ocular areaafter8-9 days postinfection of naive mice. Infectious virus was shed from the ocular tract for at least 7 days and ascending paralysis followed, leading to death of the animals between 8 and 15 days postinfection (figure 1). LD50 of $\mathrm{HSV}-1 \mathrm{KOS}$ and isolates in Balb/c mice was not performed.

\section{Discussion}

We have shown that the HSV-1 clinical isolate strain 13, 17 , 26, 39 and 42are more pathogenic in Balb/c mice than commonly used laboratory HSV-1 KOS strains and a closely related clinical isolate. In contrast to HSV-1 KOS and 27, 1, 9, 18 and 28 infections in Balb/c mice, caused poor corneal dysfunction. We cannot rule out the possibility that differences in pathogenesis phenotypes between HSV-1 KOS and 13, 17, 26,39 and 42 isolates are due they are due to the minimal or maximum mutations in these viruses that arose during their low number of in vitro passages since they originate from an active infection. However, our findings support the hypothesis that HSV-1 KOS strain differences are a determinant of disease of the phenotypes and phenotypes in addition to host response. Future studies with HSV-1 clinical isolates may yield better results on HSV-1-induced pathogenicity mechanisms (figure 1).

It is well established that minimal genetic differences in genomic skeletons of viruses have a major impact on pathogenesis. For example, the molecular basis of the highly pathogenic $\mathrm{H} 5 \mathrm{~N} 1$ influenza virus was found to be a single amino acid substitution [14]. The circulating HSV-1 strains exhibit considerable genomic variation, especially in viral glycoproteins [15, 16], even more, through the alignment performed for TK gene amplicons in different HSV1 Strains (figure 2), we could observe the lack of specificity for the identification of some variants of this virus; this situation was possibly involved in obtaining false negatives in PCR assays. The identification of new HSV-1strains and the obtaining of the sequences of their genomes will be useful for the development of effective molecular diagnostic tools that allow to solve the obtaining of erroneous results.

There is evidence of the diversity of HSV -1 , based on information obtained through restriction fragment length polymorphism (RFLP) analyses, polypeptide size, and PCR-based sequence analysis; these methodologies have made it possible to identify that HSV-1 strains vary between individuals, over sequential isolates from the same individual, and by geographic region, being observed different mechanisms involved in the development of this variation including single nucleotide polymorphisms (SNPs) and small insertions and deletions due to length variation in short sequence repeats (SSRs). As we know these variations can also affect the TK gene, and are related to its resistance to acyclovir $[16,17]$.

The identification of new HSV-1strains and the obtaining of the sequences of their genomes will be useful for the development of effective molecular diagnostic tools that allow to solve the obtaining of erroneous results $[9,16]$.

The treatment of choice for HSV-1-induced facial or ophthalmic infection, the drug is ACV. The prevalence of resistance to $\mathrm{ACV}$ is $6 \%$, mainly in immunocompromised patients and $0.3 \%$ in immunocompetents patients. In all clinical isolates the viral titer was greater than the reference strains KOS for VHS-1. In this study, we found no herpetic viral strains resistant to ACV taking an IC50 of $>3$ as a reference value. In some cases it was not possible to obtain viral amplification despite obtaining viral replication in vitro of isolates from patients with compromised immune systems or not, this could have been due to the treatments of each of them, or simply because the viral load was low, although the infection was herpetically active.

There are reports of viral strains that are highly resistant to treatment with ACV, mainly in South Africa and the United States, with differences in pathogenesis in humans.

To our knowledge, this is the first study conducted in Mexico with clinical isolates obtained from people with HSV-1 active herpetic infection. The differences that we found in response to ACV could be another viable alternative to study, because some clinical isolates were positive for TK PCR and replication negative in in-vitro culture, we thought that due to the differences of drugs this condition did not guarantees TK PCR or viral infection in cell culture.

In the year of follow-up in these same patients with a positive viral culture and who received antiviral treatment and a lack of resistance to ACV, recurrent episodes occurred, so other factors of the host intervene in the pathogenesis of the disease. There are reports associating Alzheimer's disease with HSV-1 infection. In this group of patients, antiviral 
susceptibility has not been studied, and it would be appropriate to include them in future studies.

\section{Conclusion}

This is the first study to determine ACV resistance in clinical virus isolates in Northeast Mexico. The results obtained may be useful to know the global distribution of $\mathrm{HSV}-1$ infections. Clinical resistance to ACV is related to the type of immunosuppression.

When immunosuppression is severe and is combined with long-term ACV therapy, the risk of developing strains resistant to treatment increases. Knowledge of the sensitivities of viral strains in our country and the factors involved are useful for rationalizing the use of this therapeutic tool. This study opens a line of research to determine adequate concentrations of antivirals for testing with Mexican strains.

\section{Conflicts of Interests}

The authors declare that they have no competing interests.

\section{Acknowledgements}

This study would not have been possible without our God, who gave us the strength, courage and perseverance to complete this study in favor of our Mexican society. Thanks to the staff of the Dermatology Department, and Immunovirology laboratory, of the University's Hospital, for their support during the development of this study.

\section{References}

[1] Stannard LM, Fuller AO, Spear PG. Herpes simplex virus glycoproteins associated with different morphological entities projecting from the virion envelope. The Journal of general virology. 1987; 68 (Pt 3): 715-25. Epub 1987/03/01.

[2] Mocarski Jr ES. Comparative analysis of herpesvirus-common proteins. In: Arvin A, Campadelli-Fiume G, Mocarski E, Moore PS, Roizman B, Whitley R, et al., editors. Human Herpesviruses: Biology, Therapy, and Immunoprophylaxis. Cambridge 2007.

[3] David M. Knipe, Peter M. Howley, Diane E. Griffin, Robert A. Lamb, Malcolm A. Martin, Bernard Roizman, et al. Fundamental Virology. Harvard Medical School, Boston, MA: Lippincott Williams \& Wilkins; 2001. p. 2399-459.

[4] Al-Dujaili LJ, Clerkin PP, Clement C, McFerrin HE, Bhattacharjee PS, Varnell ED, et al. Ocular herpes simplex virus: how are latency, reactivation, recurrent disease and therapy interrelated? Future microbiology. 2011; 6 (8): 877-907. Epub 2011/08/25.

[5] Irvine AR, Kimura SJ. Experimental stromal herpes simplex keratitis in rabbits. Transactions of the American
Ophthalmological Society. 1967; 65: 189-210. Epub 1967/01/01

[6] Koskiniemi M, Piiparinen H, Mannonen L, Rantalaiho T, Vaheri A. Herpes encephalitis is a disease of middle aged and elderly people: polymerase chain reaction for detection of herpes simplex virus in the CSF of 516 patients with encephalitis. The Study Group. Journal of neurology, neurosurgery, and psychiatry. 1996; 60 (2): 174-8. Epub 1996/02/01.

[7] Piacentini R, De Chiara G, Li Puma DD, Ripoli C, Marcocci ME, Garaci E, et al. HSV-1 and Alzheimer's disease: more than a hypothesis. Frontiers in pharmacology. 2014; 5: 97. Epub 2014/05/23.

[8] Wang H, Davido DJ, Morrison LA. HSV-1 strain McKrae is more neuroinvasive than HSV-1 KOS after corneal or vaginal inoculation in mice. Virus research. 2013; 173 (2): 436-40. Epub 2013/01/24.

[9] Koelle DM, Norberg P, Fitzgibbon MP, Russell RM, Greninger AL, Huang ML, et al. Worldwide circulation of HSV-2 $x$ HSV-1 recombinant strains. Scientific reports. 2017; 7: 44084. Epub 2017/03/14.

[10] Colgrove R, Diaz F, Newman R, Saif S, Shea T, Young S, et al. Genomic sequences of a low passage herpes simplex virus 2 clinical isolate and its plaque-purified derivative strain. Virology. 2014; 450-451: 140-5. Epub 2014/02/08.

[11] Kolb AW, Schmidt TR, Dyer DW, Brandt CR. Sequence variation in the herpes simplex virus $U$ (S) 1 ocular virulence determinant. Investigative ophthalmology \& visual science. 2011; 52 (7): 4630-8. Epub 2011/04/27.

[12] Morrison LA, Da Costa XJ, Knipe DM. Influence of mucosal and parenteral immunization with a replication-defective mutant of HSV-2 on immune responses and protection from genital challenge. Virology. 1998; 243 (1): 178-87. Epub 1998/04/07.

[13] Morrison LA, Knipe DM. Immunization with replication-defective mutants of herpes simplex virus type 1: sites of immune intervention in pathogenesis of challenge virus infection. Journal of virology. 1994; 68 (2): 689-96. Epub 1994/02/01.

[14] Korteweg C, Gu J. Pathology, molecular biology, and pathogenesis of avian influenza A (H5N1) infection in humans. The American journal of pathology. 2008; 172 (5): 1155-70. Epub 2008/04/12.

[15] Bowen CD, Renner DW, Shreve JT, Tafuri Y, Payne KM, Dix $\mathrm{RD}$, et al. Viral forensic genomics reveals the relatedness of classic herpes simplex virus strains KOS, KOS63, and KOS79. Virology. 2016; 492: 179-86. Epub 2016/03/08.

[16] Szpara ML, Gatherer D, Ochoa A, Greenbaum B, Dolan A, Bowden RJ, et al. Evolution and diversity in human herpes simplex virus genomes. Journal of virology. 2014; 88 (2): 1209-27. Epub 2013/11/15.

[17] Brunnemann AK, Liermann K, Deinhardt-Emmer S, Maschkowitz G, Pohlmann A, Sodeik B, et al. Recombinant herpes simplex virus type 1 strains with targeted mutations relevant for aciclovir susceptibility. Scientific reports. 2016; 6: 29903. Epub 2016/07/19. 